\title{
Gender issues in computer-supported learning
}

\author{
Cathy Gunn,* Sheila French,** Hamish McLeod,*** Mae McSporran***** and \\ Gráinne Conole****** \\ * University of Auckland, **Manchester Metropolitan University, ***University of

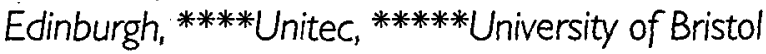 \\ email: ca.gunn@auckland.ac.nz
}

Contemporary research identifies significant gender-related differences in performance and interaction style in computer-supported learning (CSL) environments. Evidence suggests that initial perceptions of these environments as democratic and offering equal opportunities to all students were flawed because interactions that take place through electronic channels lose none of the sociocultural complexity or gender imbalance that already exists within society. This paper presents a summary of gender-related issues identified by international research and academic practice together with the opinions expressed by participants in a discussion forum staged at ALT-C in 2001. Two main questions were addressed during the conference forum. Firstly, if computer access and literacy levels are assumed to be equalizing as the literature suggests, how can educational designers using CSL technologies best serve all student groups? Secondly, does the existence of gender-based differences in behaviour and interaction style in CSL environments mean that any student group is disadvantaged? The paper concludes with suggestions about how educational designers might increase the flexibility of CSL courses to offer equal opportunities to all students. A number of issues for further research are also identified.

\section{Introduction}

In this paper, computer-supported learning (CSL) environments are defined as educational · settings where some or all communications, learning activities and administrative tasks take place through the medium of computer technology. While much research in this area focuses specifically on computer-mediated communication (CMC), we adopt a broader 
definition that also includes the use of multimedia courseware, Web resources and course management systems such as WebCT or Blackboard. An emerging term in the Australasian tertiary sector for this type of learning environment is mixed-mode education (McNaught, 2001). The critical factors are that students work in a variety of ways with computer technology and exercise a degree of autonomy that is not commonly found in lecture-based courses.

Gender-based differences in performance and interaction style in CSL environments have been recognized as an important focus for research (Herring, 1993; Weinman and Cain, 1999; Richardson and French, 2001). There are, however, conflicting views about the nature and impact of these differences and questions as to whether some student groups are disadvantaged by limited access and technology literacy remain inconclusively answered. Two citations from published sources (American Association of University Women Educational Foundation, 1998; Kleinfeld, 1998) illustrate the point of conflict:

Girls are under-represented and lower achievers in math, science and technology subjects.

Girls get higher grades, do better in standard tests and go on to achieve higher qualifications.

The critical point here is that exactly the same data were used to support both these arguments, so clearly the matter is open to interpretation.

Most early research (circa 1990) in this area suggested that women and girls had less opportunity and ability to succeed in using technology for educational purposes (McMahon, 1994; Weinman and Cain, 1999). More recently it has been found that technology access and literacy are disappearing problems and that young male students may be the group with most consistently low achievement levels in CSL (Siann, 1997; Kleinfeld, 1998). Evidence also suggests that theoretical conceptions of CSL environments as democratic and offering equal opportunities are flawed because social and educational interaction that takes place through electronic channels loses none of the sociocultural complexity or gender imbalance that exists in more traditional learning environments (Yates, 2001). Research findings from the 1990s are fairly consistent in identifying gendertypical differences in interaction styles, and in approaches to and uses of CSL technology. Many studies conclude that, as well as having less access and ability to use technology, women are further disadvantaged by dominant and sometimes even aggressive male behaviour in CMC environments. However, deeper analysis of the situation raises questions about whether the existence of such differences actually supports the common conclusion that women are disadvantaged or simply that they use technology in different ways.

This paper presents the collective experience of a small group of academics with experience of CSL in an international, though predominantly western, world context, and summarizes gender-related issues identified by contemporary research and teaching practice. The case presented here is that research findings do not support the conception that women are disadvantaged. With new educational methods and the associated forms of interaction, we may also need to look for new ways of considering identity and binary concepts such as gender. 


\section{A collaborative investigation}

This exploration of gender issues in CSL began as a collaborative venture to present a panel discussion session at the Association of Learning Technology Conference (ALT-C) in the UK in September 2001. Membership of the panel was invited on a voluntary basis and participation confirmed because of active research interests in the focus area. For the discussion session, each panel member presented a brief summary of their experience and supporting research, then concluded with a question about a particular aspect of gender difference and the implications for learning design. These questions provided the focus for small group discussions that allowed all participants to contribute their opinions and experience. The presenters' research summaries and the issues reported back by the groups form the basis of the case now presented. There is a particular focus on access and literacy issues, issues of student behaviour and cultural issues.

\section{Access and literacy issues}

Early CSL research found that women were disadvantaged by lower levels of computer literacy, access to technology and confidence in its use, and that girls were both underrepresented and lower achievers in maths, science and technology subjects at school (Weinman and Cain, 1999). These findings were open to challenge at the time (Kleinfeld, 1998), and more recent studies suggest that this is, to some extent, a disappearing problem, so that it should now be possible to examine differences that persist when imbalances due to access and literacy levels are removed.

\section{A ten-year survey of IT literacy at university entrance}

Data have bèen gathered about general levels of computer literacy among entry-level undergraduate students at the University of Edinburgh over the past ten years. The method of collection is a brief, self-report questionnaire administered through the enrolment process, and thus relatively high response rates (60-80 per cent of intake) have been obtained. The survey is believed to provide a representative example of how levels of access, attitudes and expectations about computer use have shifted over the years.

In the early years of the survey, gender was found to be a powerful predictor of the responses that students gave. In all areas of the questionnaire, gender differences were present and highly significant. Women reported themselves as less likely to own computing equipment, believed themselves to be less experienced than their male colleagues in ITrelated skills, and were generally less positive in the attitudes they expressed towards the importance and relevance of IT to their academic studies and future careers. In the early 1990 s, some of these differences were clearly related to patterns of recruitment into the various faculties of the University, and the responses were in some measure based on 'genuine' differences in the relevance of IT to academic disciplines. For example, women were more likely to be joining the Faculties of Arts and Social Sciences than Science and Engineering. Rightly or wrongly, at that time skills in computing and IT were widely seen as being more relevant to the sciences than the humanities. Over the passage of the decade, patterns of enrolment within the faculties and attitudes to IT have changed in interesting ways. While gender differences have not disappeared entirely, a definite shift is discernible.

Questions about ownership of computing equipment in the 1999 survey $(N=4360$ respondents, 75 per cent of intake) showed that women were still less likely than men to 
own a computer (see Figure 1). The data show that ownership of a personal computer is still likely to be driven, to a significant extent, by its use for the playing of games, and this is still more likely to be the preserve of men than women.

The most striking change in gender difference over the period can be seen in the responses to two questions that have been asked in identical form since the early days of the survey. Newly arriving undergraduates are asked to speculate about the frequency with which they will be expected to make use of computing technologies, and the importance which these technologies will have for their studies. In the early 1990s these questions provoked marked and consistent differences in response between male and female students. Over the decade these differences have gradually and completely disappeared (see Figure 2). These questions call for objective judgements by the students about the importance and relevance of the technologies rather than asking them to rate their personal relationship with the technologies. Male and female students have reached a point of agreement about the importance of computer technology in their work and study which augurs well for equity in applications of computers in teaching and learning.

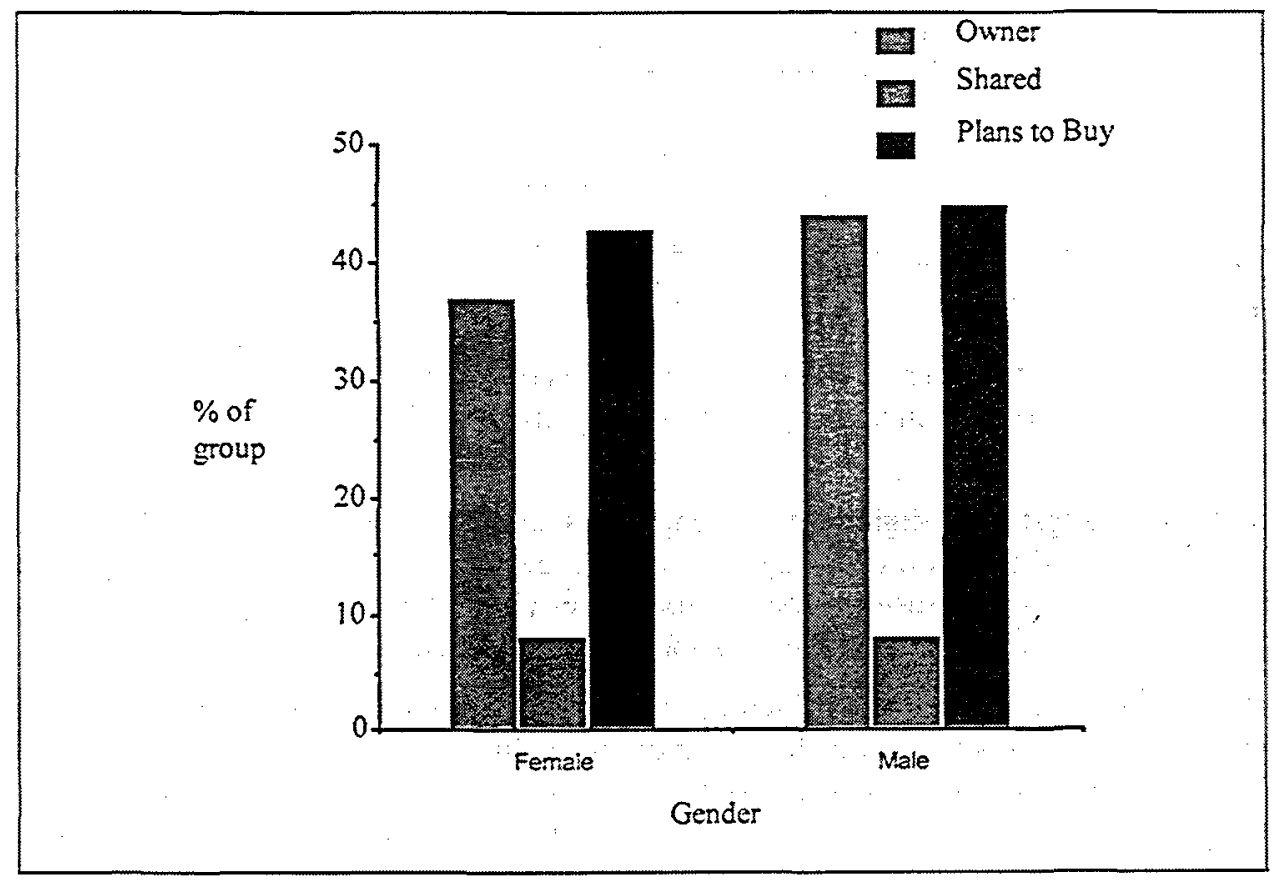

Figure 1: Ownership of, access to, and plans to buy, computing equipment for studies

Computer use patterns over the period show a marked upturn in the number of students expressing themselves as 'very confident' in their ability to use electronic mail and Webbrowsing applications. This may be explained by the changing significance of these two areas and the gender difference in confidence in these areas shows a closing gap between male and female students. The proportional increase in those describing themselves as 'very confident' proved to be greater among the women than among the men. 


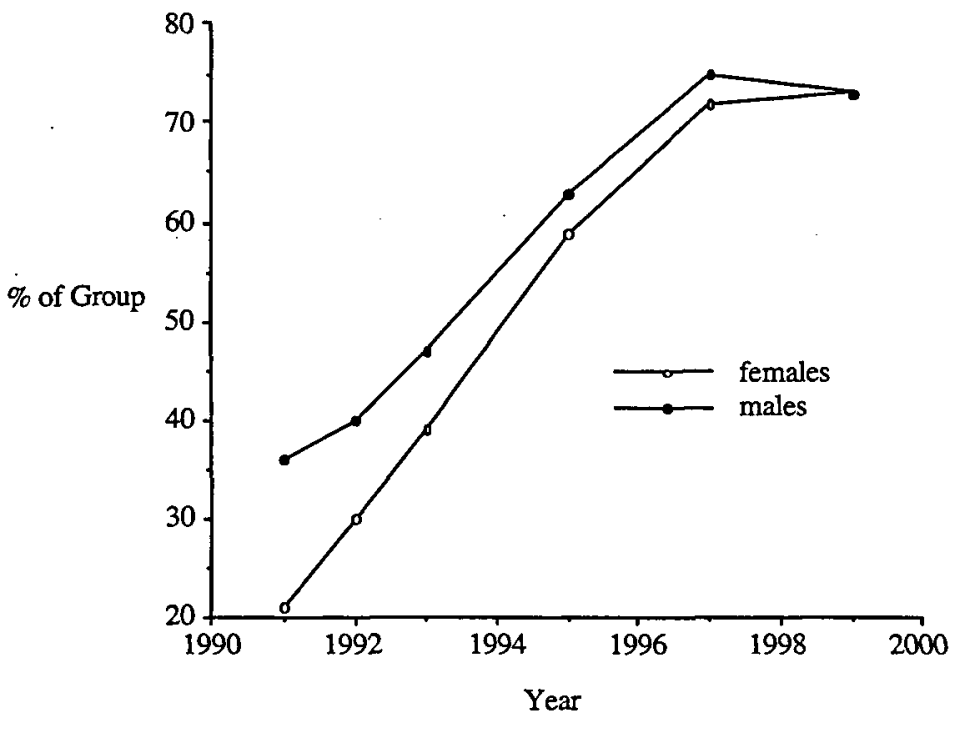

Figure 2: Percentage of the group responding either 'important' or 'vital' to the question 'How important do you think that computers will be to your university studies?'

It may be argued that women's expressed interests in computers are increasingly positive as a result of the technology's increasing pragmatic significance. This point is summed up in a recent article in the New York Times:

Grown-ups' angst over digital gender gap looks quaintly irrelevant now that teenage girls are addicted to instant messaging and the majority of Internet users in the States are female. Girls had no trouble adapting to computers once the machines did something that interested them. (New York Times, 5 August 2001)

The assumption that gender-based inequity in terms of access and computer literacy is a disappearing problem is borne out by the findings of this study along with other research and reports from various sources across the western world. The focus of attention now turns to the differences that persist in this 'more equal' environment and possible interpretations of their impact.

\section{Disadvantaged or just different?}

Once male and female students are more or less equal in terms of access and literacy, experience shows that gender-based social relationships, interaction styles and inequities that exist in traditional learning situations correspond fairly closely to those found in CSL environments (Herring, 1993). These evidence-based assumptions contradict more optimistic claims that technology is gender-neutral and that it provides a democratic and equal environment. 
A number of researchers have identified common differences in the behaviour of male and female students in CSL environments (Richardson and French, 2001; We, 1993; Everts, 1998; Barrett and Lally, 1999). These differences include self-reported levels of confidence in ability to work successfully with technology, use of support systems and patterns of interaction. At a deeper level of analysis, studies of dialogue in CMC consistently reveal gender differences in styles of participation and contribution. Generally speaking, women talk less, contribute less frequently, do not receive positive feedback to their contributions and do not appeal to the same sources of support as their male counterparts (Richardson and French, 2001; Herring, 1993). However, there is some debate over whether these issues constitute disadvantage or simply differences in interaction styles. There is evidence of male behaviour patterns that could be described as dominant in discussion areas (Herring, 1994) and a notable tendency for men to espouse more confidence in their general capabilities in CSL environments than do women. However, there is no evidence that this leads to better performance or learning outcomes and some research has found the opposite to be true (Richardson and French, 2001; McSporran and Young, 2001). Two case studies will now be presented in order to demonstrate how similar courses offered in different locations, the UK and New Zealand, reached this conclusion. The case studies involve tertiary-level IT modules with a significant degree of flexibility in study mode and method because of the mixed-mode teaching approach of CSL environments.

\section{Case Study I: Electronic Information Services}

This case study involves a cohort of Information Technology (IT) undergraduates taking a first-year module called 'Electronic Information Services' (EIS) offered by the Information Systems Institute at the University of Salford in 1999 (see Richardson and French, 2001 for a fuller report). Whilst the course materials were presented mainly online, students attended some lectures and had access to paper-based materials. From a class of 140,70 per cent of the students were male.

The course designers were well aware of gender differences in attitudes to technology. Recent research showed a decline in women taking IT subjects at university (Siann, 1997), and that the IT industry in the UK was male-orientated with women representing just 22 per cent of those in the profession (NCET, 1996). The claims that men have the monopoly on technology (Wajcman, 1991) and that women may even be excluded from its use by virtue of its design and function (Griffiths, 1985) are important issues to confront when using technology in learning. Given this background there was some concern that women might even shun the use of computers in learning.

\section{The research process}

The study was carried out using formative and summative questionnaires to collect quantitative and qualitative data. A number of meetings with focus groups also took place. Further information was gleaned from the experiences of the lecturer and notes kept in the form of a diary. The research focused on gender issues in the following key areas:

Flexibility and access

The study showed that all students appreciated the flexibility of the module and liked to be able to work at their own pace. All students used computers at the university. Most also had access to computers in the home, although females reported more problems with access such as having to share the computer with other family members or friends. Several 
females reported that they did not have priority access to the computer in the home, whereas none of the males reported this. Kirkup and Abbot (1997) found that female students regarded a computer in the home as a family resource and did not see their studies as a reason for priority access. This situation is common to other studies and may warrant further investigation.

\section{Confidence}

All students used a mix of online and paper-based materials. Whereas males were very confident that they would enjoy using the online materials, several women stated they were apprehensive about using the materials and about their overall ability in the technical aspects of the course, that is, designing Web pages.

\section{Approaches to learning}

Gender differences emerged in learning. The female students spent much more time preparing for the module. This could be because of their general lack of confidence. The frequency with which students studied at home differed according to gender; whilst most males reported mainly working between the hours of 4 and 8 p.m., the majority of women studied later in the evening, fitting study around other commitments such as family.

\section{Support}

Gender differences emerged in the support mechanisms utilized by students. Women mainly emailed the lecturer and friends whilst males reported contact with their peers at University as well. No women seemed to use this method of support and this raised the issue of whether this type of learning isolated female students. There is conflicting evidence on this subject. Some research has found that women, especially those with family commitments, embrace the flexibility in learning schedules and attendance requirements which online learning brings (Von Prummer, 1994). Parallel research has produced conflicting evidence suggesting that women are 'social learners' and prefer traditional learning over the need to adapt to another imposed model such as online learning (Von Prummer, 1994; Kirkup and Abbot, 1997). The researchers in this case felt that this could be another important issue for further study.

\section{Learning outcomes}

The confidence that the males expressed and the apprehension of many of the women were not substantially reflected in the grades they achieved. The males achieved slightly higher grades than the women as a whole, on what might be deemed the more technical aspect of the module (designing Web pages). Overall, however, the women's marks were concentrated at the top end of the grades for all of the.assessed work.

Implications for learning with technology

From this research it can be concluded that the online learning experience may well be different for males and females. The females in this study seemed to have less confidence than their male counterparts and less guaranteed access to technology. In order to ensure equality, the needs are to improve access, make sure learners are not isolated and find ways of instilling confidence in some of the female students. Social issues such as ownership of technology are at the forefront of research and need further discussion. The issues are complex and, as the other studies in this paper demonstrate, there is much conflicting evidence. 


\section{Case study 2: Interactive Web Design}

Research conducted in a similar context in a New Zealand tertiary institution found that female students performed consistently better than males in online courses (McSporran, Dewstow and Young, 1999; McSporran and Young, 2001). A course in Interactive Web Design enrols 70-100 students per semester and options are available to complete the course entirely online or to attend weekly classroom sessions in addition to independent study. The course structure and assessment have remained more or less the same over the three-year period of the study and involve:

- a balance of theory and practical exercises;

- self-test quizzes;

- access to readings, URLs and FAQs;

- constant feedback from tutors;

- a research project requiring team collaboration and report preparation;

- a Website development assignment and a final exam.

Knowledge of contemporary research findings together with the benefits of past experience provided the basis for initial assumptions that female students might perform better on the research assignment because it involved communication and teamwork, while males may do better on the technical task of Website development. However, these assumptions proved to be unfounded as the female students performed consistently better than males on both tasks. Analysis of course-entry questionnaires showed that females rated themselves less competent with Internet skills and experience at the outset, a situation that is reflected in the findings of other research (Yates, 1992). Analysis of participation in online activities recorded by the course management system server then raised the possibility that male students may be overconfident in their ability and think they do not have to put in as much effort to meet the course requirements (see Table 1). This analysis revealed the following characteristics of male student behaviour:

- greater non-submission of a complete assignment;

- greater non-completion of all parts of each assignment;

- lower number of online quiz attempts;

- lower course Website page views;

- lower bulletin board participation.

Although these figures are relatively crude measures of cause and effect regarding performance levels, the tentative conclusion is that the course works well for women and mature students, while the younger male students appear to need more motivation and discipline than weekly classroom sessions currently provide. The reasons for better performance of women, and in this case mature students, are believed to include stronger motivation to succeed, greater ability to work independently and self-manage multi-tasking lives. These case-specific findings are further supported by a Canadian study that reports opportunities for communication and participation in course-related activities being 


\begin{tabular}{llc}
\hline Semester & Female \% & Male \% \\
\hline Quiz attempts & & \\
$\quad$ No of quizzes attempted & 11.4 & 7.4 \\
Never attempted a quiz & 4.0 & 20.0 \\
Bulletin Board Participation & & \\
$\quad$ Posts read & 38.5 & 16.2 \\
Posts made & 4.3 & 2.6 \\
\hline & Female $(n)$ & Male $(n)$ \\
\hline Total page views & 416.9 & 249.2. \\
\hline
\end{tabular}

Table I: System data of male and female student participation in CSL elements

enhanced for women when they do not have to enter 'inhospitable' environments such as computer labs (Chegwidden, 2000).

It seems then, that although a significant body of literature suggests that suitable educational models are currently not widely used to promote women-friendly CSL environments, some situations are not particularly male-friendly either. The challenge is to find solutions that offer equal opportunities to all students. The questions that this raises are what characteristics make learning environments friendly to both male and female students, and how can opportunities to participate and succeed be enhanced for all. Before presenting the solutions proposed by the discussion groups, the impact of culture on gender differences is considered worthy of mention.

\section{Cultural impact on gender relations}

A review of international literature (notably from the US, Europe and Australasia) on gender, identity and performance issues in CSL environments raises questions about how far the differences are culturally determined (Barrett and Lally, 1999; Eubanks, 2000). Some variation may be attributable to the style of report or the nature of the gender imbalance in particular situations. However, it may also be the case that culturally based metaphors significantly influence perceptions of technology environments, their suitability for different groups, the values they reflect and the behaviour that is acceptable within their confines.

The term 'cyberspace' itself has some significance in a discussion of cultural influence on gender issues. The concept of cyberspace has its origins in a science fiction work, Neuromancer, published by Gibson in 1984. Gibson's cyberspace is a female region used and controlled by men; it is also highly sexualized. Women in this region are simply resources, not active agents or users of technology. So the very metaphor that is used to define the online 'space' in which students now interact has its roots in a very unequal society.

In contemporary cultures, there do appear to be differences that could be attributed to the sociocultural contexts in which educational experiences are studied and reported. 
Published studies from the southern US identify dominant and sometimes even deviant behaviour among males (Herring, 1994; Blumenstyk, 1997; Blum, 1999 Herring, 1999). For example, Blum found that males talk more and interpreted this as controlling behaviour, noted that they used different tones, ones of arrogance and certainty that she interpreted as intimidating to women, and asked fewer questions which implied that they had more knowledge. She concluded that interactions in CMC reflect those of face-to-face culture. Although the findings of this study could be interpreted quite differently and the methodology questioned because of the small sample used for a study that claims generalizable results, the fact that the author made these interpretations may say something about the culture in which she works. Herring reported construction and maintenance of asymmetrical gender/power dynamics and exposed blatant double standards in freedom of speech 'rhetoric' (Herring, 1999).

Moving to a different cultural environment, Barrett and Lally (1999) present a case study from the UK in which male and female students played notably different roles:

- males talk more;

- meta/cognitive content was the same;

- social content was higher for males;

- interaction was higher for females;

- only females had persistent access problems.

Although some findings are common with the US studies, the researchers do not draw conclusions about women being disadvantaged. Rather they seek to explore the meaning of these differences in terms of perceived user friendliness of the environment and its impact on student performance.

In the other cultural environments covered by this study, requests for participation in this research from Australian academics resulted in expression of the opinion that 'gender is simply not an issue worthy of attention in this country'. In New Zealand, the only research available suggests that women are performing better than their male counterparts, although this may reflect a lack of investigation (Gunn and Barnett, 2001) and published research rather than a difference in what appears to be the norm elsewhere. The conclusion here is that broader aspects of local culture may indeed be reflected in the interaction styles of students in CSL environments. To a great extent, these issues go beyond the scope of influence of educational designers although they should be afforded due consideration in learning design.

\section{Conclusions and recommendations}

Consensus reached by the discussion groups at ALT-C was that the differences identified by the presenters and supported by contemporary research exist in most western world educational environments and present significant challenges to educators using new technologies in teaching. However, a range of interpretations of both the underlying causes and the implications of these differences is possible. The critical issue is to develop widely applicable solutions rather than conducting more in-depth research into the causes. 
It was agreed that inequality in levels of access and computer literacy among student populations are disappearing problems, and that male and female users generally take different approaches to the use of technology, that is, exploratory and developmental versus practical and instrumental. This inherent difference in approach sparked a debate about whether some aspects of gender are in fact attributable to sociocultural construction or to biological difference. Regardless of the outcome of that particular debate, the implications for CSL designers are that they have to accommodate a range of approaches to the use of technology and design learning environments that do not implicitly favour any particular approach. This is considered to require a degree of flexibility that many online environments currently do not include. For example, educationally focused games and challenges may be more appealing to a typically competitive male orientation, while communicative and collaborative activities may appeal more to typical female styles. The usefulness of these types of activities will depend on suitability to discipline and subjectrelated learning objectives as well as to gender-typical approaches.

A critical issue in encouraging equal participation by both genders may be the levels and types of support provided for learners. For example, good moderation in discussions can help to ensure equal and appropriate levels of participation. Inclusion of opportunities for self-assessment during a course can support self-monitoring of performance to ensure that espoused confidence is backed up by the ability to attain high performance levels with or without specifically requested support. The issue of confidence is perceived to be a particular challenge for male students who are considered less likely to ask for assistance as well as less likely to recognize the need to participate in all course-related activities and preparations for assessment. It was also noted, however, that in a context characterized by rapid change in so many areas - technology, educational theories, design of learning environments, student demographics and professional requirements - these issues are dynamic and in need of constant reappraisal. Issues of gender should not be treated in isolation from other factors such as learning styles, educational backgrounds and capabilities.

A more general point was also raised about computer-based course management systems in their current incarnation being predominantly designed to support didactic rather than constructive learning experiences. This is one more issue that needs to be addressed in the design of learning environments that offer equal opportunities to all students. It was generally concluded that gender is just one of many areas of focus for research and development of effective CSL environments and activities.

\section{References}

American Association of University Women Educational Foundation (1998), Gender Gaps: Where Schools Still Fail Our Children, Washington DC: AAUWEF.

Barrett, E. and Lally, V. (1999), 'Gender differences in an on-line learning environment', Journal of Computer Assisted Learning, 15, 48-60.

Blum, K. D. (1999), 'Gender differences in asynchronous learning in higher education: learning styles, participation barriers and communication patterns', Journal of Asynchronous Learning Networks, 3 (1), 46-66. Available from: www.aln.org/alnwebl journalljaln-vol3issue1.htm 
Blumenstyk, G. (1997), 'A feminist scholar questions how women fare in distance education', The Chronicle of Higher Education. Available from: http://chronicle. com/colloquy/97/distancelbackground.htm.

Chegwidden, P. (2000), Feminist Pedagogy and the Laptop Computer. Women, Work and Computerization: Charting a Course to the Future, Vancouver, BC: Kluwer Academic Publishers, 293-9.

Eubanks, V. (2000), 'Paradigms and perversions: a woman's place in cyberspace', PCSR Newsletter, 18 (1). Available from: http://www.cpsr.org/publications/newsletters/issues/2000/ Winter2000leubanks.html

Everts, S. (1998), Gender and Technology: Empowering Women, Engendering Development, London and New York: Zed Books.

Gibson, J. (1984), Neuromancer, New York: Ace Books.

Gunn, C. and Barnett, J. (2001), 'On-line learning: a quality experience', in P. Roberts and M. Chambers (eds), Digital Developments in Higher Education, Los Angeles, CA: Taylor Graham, 139-60.

Griffiths, D. (1985), 'The exclusion of women from technology', in W. Faulkner and E. Arnold (eds), Smothered by Invention, London: Pluto Press, 51-71.

Herring, S. (1999), 'The rhetorical dynamics of gender harassment on-line', The Information Society, 15 (3), 151-67.

Herring, S. (1994), 'Gender differences in computer mediated communication: bringing familiar baggage to the new frontier', in Making the Net*Work*: Is There a Z39.50 in Gender Communication?, Miami: American Library Association. Available from: http://cpsr.org/cpsr/gender/herring.txt.

Herring, S. (1993), 'Gender and democracy in computer mediated communication', Electronic Journal of Communication, 3 (2), 1-17. Available from: http://www.cios.org/ wwwlejclv3n293. htm

Kirkup, G. and Abbot, J. (1997), The Gender Gap. A Gender Analysis of the 1996 Computing Access Survey, PLUM Paper number 80. Programme on Learner Use of Media, Milton Keynes: Open University.

Kleinfeld, J. (1998), The Myth That Schools Shortchange Girls: Social Science in the Service of Deception, Washington DC: The Women's Freedom Network.

McMahon, P. (1994), Strategies to Facilitate Student Computer Usage: Are Attitudes the Answer?, Survey Report, Belfast: Queen's University.

McNaught, C. (2001), 'Quality assurance for online courses: from policy to process to improvement?', Paper presented at the Ascilite 2001: Meeting at the Crossroads, Melbourne, Vic.

McSporran, M., Dewstow, R. and Young, S. (1999), Who Wants to Learn On-line? Identifying Our Flexible Learners, Proceedings of the World Conference on Educational Multimedia, Hypermedia and Telecommunications, Washington: Association for the Advancement of Computing in Education. 
McSporran, M. and Young, S. (2001), 'Does gender matter in online learning?', Association of Learning Technology Journal, 9 (2), 3-11.

NCET (1996), Gender and IT, Coventry: NCET.

Richardson, H. and French, S. (2001), 'Education on-line: what's in it for women?', in Women, Work and Computerization: Charting a Course to the Future, Vancouver BC: Kluwer Academic Publishers.

Siann, G. (1997), 'We can, we don't want to: factors influencing women's participation in computing', in R. Lander and A. Adam (eds), Women in Computing, Exeter: Intellect Books.

Von Prummer, C. (1994), 'Women-friendly perspectives in distance education', Open Learning, 9 (1), 3-12.

Wajcman, J. (1991), Feminism Confronts Technology, Cambridge: Polity Press.

We, G. (1993), Cross-gender Communication in Cyberspace, Department of Communication, Burnaby, BC: Simon Fraser University.

Weinman, J. and Cain, L. (1999), 'Technology - the new gender gap', Technos, 8 (1), 9-12.

Yates, S. J. (1992), Gender and Computer-mediated Communication: An Analysis of DT200 in 1990, CITE Report No 158, Milton Keynes: Open University.

Yates, S. J. (2001), 'Gender, language and CMC for education', Learning and Instruction, $11,21-34$. 